\title{
The Ultra-Cold Polarized Hydrogen Jet Project
}

\author{
R.S. Raymond, D.G. Crabb, T. Roser, J.A. Stewart \\ Randall Laboratory of Physics, The University of Michigan, \\ Ann Arbor, Michigan 48109 \\ G.R. Court \\ Department of Physics, Massachusetts Institute of Technology \\ Cambridge, Massachusetts 02139
}

\begin{abstract}
Research is described on a jet of polarized atomic hydrogen. Atoms in the two lower hyperfine states are trapped at $0.4 \mathrm{k}$ in a $5 \mathrm{~T}$ magnetic field. Microwaves at $140 \mathrm{GHz}$ then drive transitions to the upper hyperfine states and these atoms are expelled from the field to form the beam. Atoms have been trapped, transitions driven, and atoms detected outside the trapping field, but preliminary measurements are not fully understood. Studies are continuing.
\end{abstract}

Work is continuing to test the applicability of cryogenic atomic physics techniques to the production of dense polarized atomic hydrogen beams. At high magnetic fields $(5 \mathrm{~T})$ and low temperatures $(0.5 \mathrm{k})$, the magnetic energy of a hydrogen atom, approximately $\mu_{e} \cdot \mathrm{B}$, is considerably higher than the thermal energy, $\mathrm{kT}$. When cold atoms approach a high magnetic field, atoms in the two upper hyperfine states see a potential hill and are repulsed. Atoms in the two lower hyperfine states see a potential well, are attracted in, thermalize by hitting cold walls and are trapped. These trapped atoms are then irradiated with microwaves of the proper frequency (approximately $140 \mathrm{GHz}$ for a $5 \mathrm{~T}$ field) to drive transition to one of the upper hyperfine states. These flipped atoms, now on a potential hill, are accelerated out of the field to form the beam.

This scheme is attractive in that the production rate of polarized atoms should be very high and, since cold atoms are accelerated, the velocity spread should be relatively small. With this good beam quality we hope to be able to focus the beam to produce a target with a thickness of $10^{14} / \mathrm{cm}^{2}$ perpendicular to the jet direction.

The apparatus to test these ideas is shown in Figure 1. The vertical section consists largely of parts of a high-power dilution refrigerator. The mixing chamber is horizontal, inside a high-uniformity superconducting solenoid. Atoms are produced from molecules in a liquid nitrogen-temperature discharge in the vertical section, and then flow down a teflon transfer tube until they emerge to see liquid helium-coated walls cooled by the final heat exchanger of the refrigerator. At these sub-kelvin temperatures, all surfaces must be coated with helium to prevent recombination. The cooled atoms then see the fringe field of the solenoid, where electron-spin selection takes place and atoms in the lower states are drawn into the field. Microwaves enter from the left on the figure and atoms leaving toward the right are detected. 
We drive only one of the two allowed hyperfine transitions at a time, populating only one of the upper states. We expect the output beam to include atoms in both states, however, because of spin-exchange collisions between flipped and unflipped atoms. This combination of states could result in a decrease in proton polarization, but full proton polarization can be recovered by using the $\mathrm{rf}$ transition techniques which are standard in polarized ion sources.

To detect hydrogen atoms expelled by the field we have looked at the temperature rise of a light copper detector caused by the recombination of atoms on the surface. Some results are shown in Figure 2. In this case, the microwave frequency and power (about $15 \mathrm{~mW}$ ) remained constant and the magnetic field was ramped up. The spacing between the peaks agrees with the spacing between the two allowed hyperfine transitions. Measurements have also been made leaving the field fixed and pulsing the microwaves with a mechanical switch. We have some qualitative understanding of the line shape, but we don't understand the detector efficiency well enough to know how much beam was expelled. Our program of measurements has also been hampered by instabilities which sometimes occur in the helium film which covers the mixing chamber walls. We believe that these instabilities are due to a build-up of $\mathrm{H}_{2}$ snow in the low-temperature section of the accommodator and we are now rebuilding the feed system to eliminate this problem. We are also considering other diagnostic tools to measure hydrogen intensity, velocity, and polarization. These include a compression tube, differential focussing, and perhaps a maser.

Finally, we are considering the implications of running DC, as needed for a storage ring, instead of pulsed as originally planned. Running in a pulsed mode we would store atoms until a density of about $10^{16} / \mathrm{cm}^{6}$ is reached. A pulse of microwaves would then produce the beam. Calculations indicate that higher storage densities would result in unacceptably high losses of flipped atoms to recombination. For DC operation we want, effectively, an active spin filter instead of a storage cell. This would be achieved by having high enough microwave fields that atoms are flipped soon after entering the high field. The resulting low density allows much greater freedom in choosing wall temperatures and geometry than the high density needed for the pulsed mode.

We would like to thank A.D. Krisch, D. Kleppner, and T.J. Greytak for continued considerable support and advice. This work has been supported by the U.S. Department of Energy. 


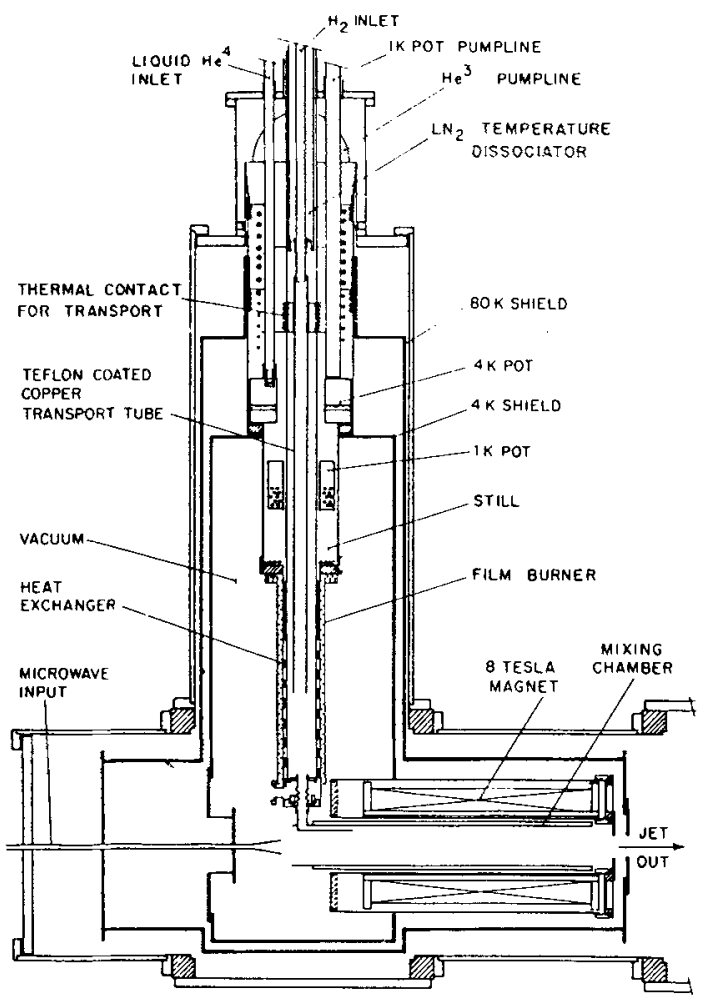

Fig. 1. JET TEST APPARATUS

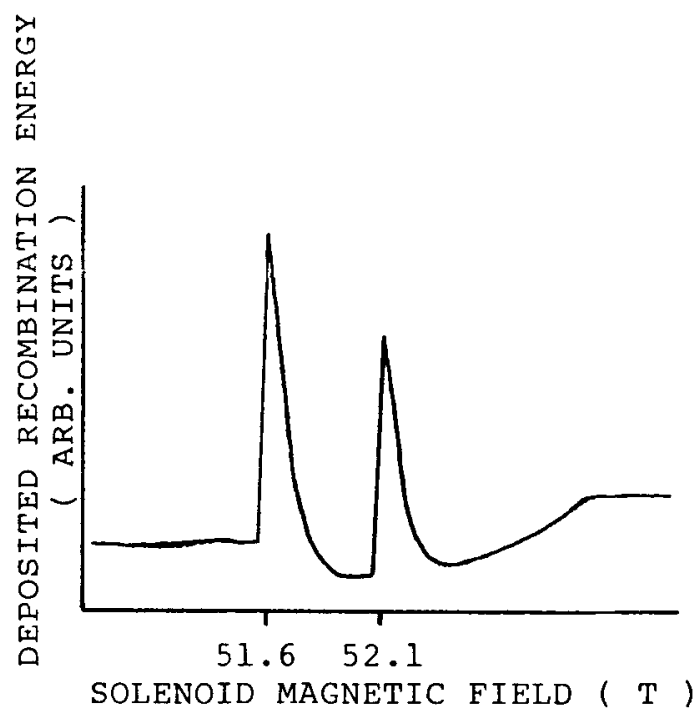

Fig. 2. Detector response for ramped magnetic field 